\title{
Lagrangian chaos and multiphase processes in vortex flows
}

\author{
Thomas H. Solomon", Brian R. Wallace, Nathan S. Miller and Courtney J. L.. Spohn \\ Department of Physics, Bucknell University, Lewisburg, PA 17837
}

\begin{abstract}
We discuss experimental and numerical studies of the effects of Lagrangian chaos (chaotic advection) on the stretching of a drop of an immiscible impurity in a flow. We argue that the standard capillary number used to describe this process is inadequate since it does not account for advection of a drop between regions of the flow with varying velocity gradient. Consequently, we propose a Lagrangiangeneralized capillary number $C_{L}$ number based on finite-time Lyapunov exponents. We present preliminary tests of this formalism for the stretching of a single drop of oil in an oscillating vortex flow, which has been shown previously to exhibit Lagrangian chaos. Probability distribution functions (PDFs) of the stretching of this drop have features that are similar to PDFs of $C_{L}$. We also discuss on-going experiments that we have begun on drop stretching in a blinking vortex flow.
\end{abstract}

* Author to whom correspondence should be sent: Department of Physics, Bucknell University, Lewisburg, PA 17837 USA; email: tsolomon@ @ucknell.edu; phone: 1-570-577-1348; fax: $1-570-577-3153$

\section{Introduction}

For the past couple of decades, it has been well-understood that fluid elements in wellordered laminar flows can follow trajectories that are chaotic in the sense that nearby tracers separate exponentially in time ${ }^{1,2}$. This effect - referred to as Lagrangian Chaos or chaotic advection - can result in significant enhancements in local mixing of impurities as well as longrange transport. Less attention has been given however to the importance of Lagrangian chaos when discussing dynamical processes occurring in fluid flows.

In this article, we describe studies of the effects of Lagrangian chaos on multiphase fluid processes. In particular, we examine the importance of Lagrangian effects in the stretching and breakup of drops of an immiscible impurity as they are advected through a flow. We define a Lagrangian-generalized Capillary number that is based on finite-time Lyapunov exponents. We 
then present preliminary results of experimental and numerical studies for the stretching and breakup of drops of oil floating on the surface of a chain of oscillating vortices ${ }^{3,4}$, a flow whose mixing properties have been well-studied ${ }^{5,6,7}$. Statistics of the stretching of the drop are compared with those of the Lagrangian capillary number.

In Section II, we review the basic theory commonly used to describe drop stretching in a flow, and present a generalized approach that incorporates the Lagrangian framework into the analysis. In Section III, we describe the oscillating vortex flow and explain the experimental and numerical techniques used in these studies. Experiments on the stretching and breakup of oil drops in the flow are discussed in Section IV, along with numerical simulations of the finite-time Lyapunov exponents. Preliminary studies of droplet stretching in a blinking vortex flow are presented in Section V. We discuss the results and conclude in Section VI.

\section{Drop-stretching and breakup: Eulerian versus Lagrangian approaches}

There are two main frameworks for analyzing the effects of fluid flows on processes - an Eulerian framework and a Lagrangian framework. The Eulerian framework is based on snapshots of the velocity field describing a fluid flow. In the Lagrangian framework, however, an impurity or fluid element is followed as it moves through the flow. Of course, the motion of an impurity can be determined from the Eulerian framework by treating the velocity field as a set of differential equations that can be integrated to determine the trajectory, so the two frameworks are not independent. However, the Lagrangian framework can reveal complexity that would not be expected from the velocity field itself. The most salient example of this is Lagrangian chaos (or chaotic advection), where a tracer in a simple, laminar, well-ordered velocity field might follow chaotic trajectories in the sense that nearby tracers separate exponentially in time.

The stretching and breakup of drops of immiscible impurities is a subject that has been studied extensively by other investigators ${ }^{8,9}$. However, almost all of those studies have analyzed the phenomena from an Eulerian perspective. But not only is a drop stretched by its surrounding flow, it is also advected to different regions which may have significantly different velocity gradients. Our assertion is that the Lagrangian framework, in fact, is the more natural framework for studying these processes.

We begin by reviewing the standard (Eulerian) description used for drop stretching, after which we introduce a generalization to account for advection.

\section{A. Droplet stretching and breakup in Eulerian framework}

When discussing the behavior of immiscible impurities in a flow, a Capillary number is defined $^{8,9}$ as follows (see Fig. 1):

$$
C=\frac{\mu G a}{\gamma}
$$

where $\mu$ is the viscosity of the drop, $G$ is the velocity gradient experienced by the drop, $a$ is the typical radius of the drop, and $\gamma$ is the surface tension between the drop and the surrounding fluid. The capillary number can be thought of as a measure of the competition between two 


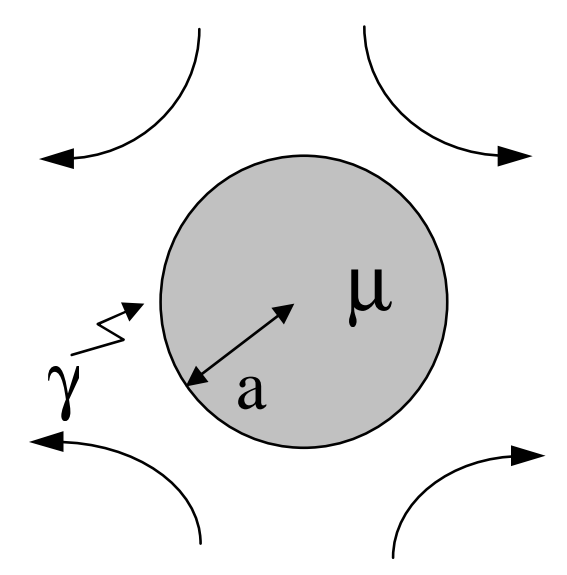

Figure 1. Sketch showing the symbols used in the Eulerian definition of the Capillary number. The typical radius and viscosity of the drop are denoted by $a$ and $\mu$, respectively, and $\gamma$ denotes the surface tension between the drop and the surrounding fluid. The arcs denote an extensional flow in which the drop is held.

effects - the tendency of the flow to stretch a drop into an elongated shape, and the tendency of the surface tension to minimize surface area and pull the drop back to a circular shape. From this perspective, the Capillary number can be rewritten as a ratio of time scales:

$$
C=\frac{\tau_{c}}{\tau_{f}}
$$

where $\tau_{c}=\mu a / \gamma$ is the capillary time scale and $\tau_{f}=G^{-1}$ is the velocity time scale.

Given a ratio between the viscosity of the drop and the surrounding fluid, the magnitude of $C$ determines the behavior of the drop. We can characterize the elongation of the drop by the quantity

$$
\xi=\frac{r_{\max }}{r_{\min }}-1
$$

where $r_{\max }$ and $r_{\text {min }}$ are the maximum and minimum distances from the drop center to its perimeter. (For small elongation, the drop is an ellipse and $\xi$ reduces to the eccentricity of that ellipse.) If $C$ is small, the surface tension dominates and the drop remains close to a circular shape. As $C$ is increased, the flow becomes more and more significant and the drop stretches more and more, with $\xi$ growing monotonically with $C$. If $C$ exceeds a critical value $C_{c}$, then the drop breaks up into a collection of smaller droplets ${ }^{9}$.

There is an assumption in this approach: namely, the drop needs to stay in the same region of the flow throughout the processes to maintain constant $G$ and therefore constant $C$. In fact, experiments that have been done to test these theories have typically been performed with feedback systems that hold the drop in the same region. ${ }^{10}$ 
In nature, drops do not usually stay in one place in a fluid flow, especially in the vicinity of a hyperbolic fixed point (such as in the extensional flow of Fig. 1) where the stretching of the drop is most pronounced. Here is where the Lagrangian framework comes into play. In a real flow, a drop does not just sit in one place experiencing just one velocity gradient. Rather, it moves around the flow, between regions of varying shear. Clearly, the capillary number as defined here is not sufficient in general; at the very least, something has to be done about the "G" in the numerator.

\section{B. Lagrangian-generalized capillary number}

Ultimately, the standard capillary number has to be replaced with a generalized "Lagrangian capillary number" that accounts for the effects of advection. In this manner, the movement of the drop between regions of varying shear can be accounted for. This is particularly important in light of the possibility of Lagrangian chaos in laminar fluid flows.

The time scale $\tau_{f}$ which characterizes the tendency of the flow to stretch the drop needs to be replaced by a Lagrangian timescale, something that takes into account the fact that how much a drop stretches depends not just on the velocity gradient that the drop is experiencing now, but rather on a Lagrangian average of the strain experienced by the drop during its motion. A natural choice is the inverse $\lambda^{-1}$ of the largest Lyapunov exponent, defined by the relation

$$
\lambda_{\max }=\lim _{t \rightarrow \infty}\left(\frac{\ln \left(\Delta(t) / \Delta_{0}\right)}{t}\right)
$$

where $\Delta_{0}$ is the initial separation of two nearby passive tracers in the flow, and $\Delta(\mathrm{t})$ is the separation of the same two tracers after a time $t$.

This is not the complete story however. As defined here, the Lyapunov exponent effectively averages over the entire motion of the drop, but the drop has only a finite memory. The time scale for this memory is just the capillary time scale $\tau_{\mathrm{c}}$. So, instead of the standard Lyapunov exponent, we use the finite time Lyapunov exponent

$$
\lambda(\tau)=\frac{\ln \left[\Delta(\tau) / \Delta_{0}\right]}{\tau}
$$

and use the capillary time $\tau_{\mathrm{c}}$ to set the duration of this finite time. The result is a Lagrangian capillary number

$$
C_{L}=\tau_{c} \lambda\left(\tau_{c}\right)=\frac{\mu a \lambda\left(\tau_{c}\right)}{\gamma} .
$$

Note that this Lagrangian capillary number reduces to the Eulerian capillary number in the limit where $\tau_{\mathrm{c}} \rightarrow 0$ since $\lambda\left(\tau_{\mathrm{c}} \rightarrow 0\right)=\mathrm{G}$. This makes sense, because in the limit where $\tau_{\mathrm{c}} \rightarrow 0$, the drop has no memory, and therefore the stretch of this drop should be dependent only on the local velocity gradient. 
Given this proposed definition for a Lagrangian-generalized capillary number, the magnitude of $C_{L}$ - which is now a time-varying quantity since it changes as the drop moves through the flow - would be expected to be related monotonically to the stretch of the drop $\xi$. And since $C_{L}$ is proportional to the finite-time Lyapunov exponent (which also varies in time), a drop moving through a flow would be expected to be a probe of finite-time Lyapunov exponents; specifically, the elongation of the drop at any time would be a measure of $\lambda\left(\tau_{c}\right)$.

One of the goals, then, of our experiments is to test these ideas by monitoring the elongation $\xi$ of a drop as it moves through a flow, and compare the statistics of $\xi$ to those of the finite-time Lyapunov exponents and the Lagrangian-generalized capillary number.

Note that if this definition of $C_{L}$ is appropriate, then it is significant even if there is no Lagrangian chaos. Technically, Lagrangian chaos requires a positive (infinite-time) Lyapunov exponent. But finite-time Lyapunov exponents can be positive even in the absence of any deterministic chaos.

\section{Numerical and experimental methods}

The primary flow studied is an oscillating vortex chain as shown in Fig. 2. The flow can be either time-independent or periodic; in the latter case, the entire velocity field oscillates laterally. The velocity field can be modeled by applying Hamilton's equations of motion to the following streamfunction ${ }^{11}$ :

$$
\psi(x, y, t)=\frac{A}{k} \sin \{k[x+B \sin \omega t]\} W(y),
$$

where $B$ and $\omega$ are the amplitude and angular frequency of the lateral oscillation, and $W(y)$ is a function that accounts for the boundary conditions. (From hereon, we will use the symbol $b=$

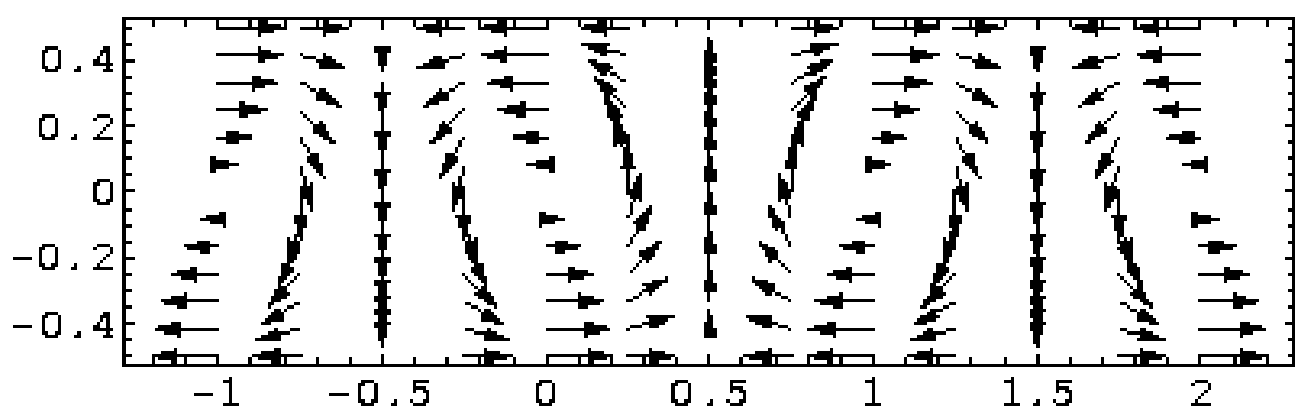

Figure 2. Sketch of alternating vortex chain used in these studies. When time-periodic, the entire flow oscillates laterally. This sketch corresponds to the case with free-slip boundary conditions. The no-slip case looks qualitatively similar, although the velocities go to zero at the top and bottom. 


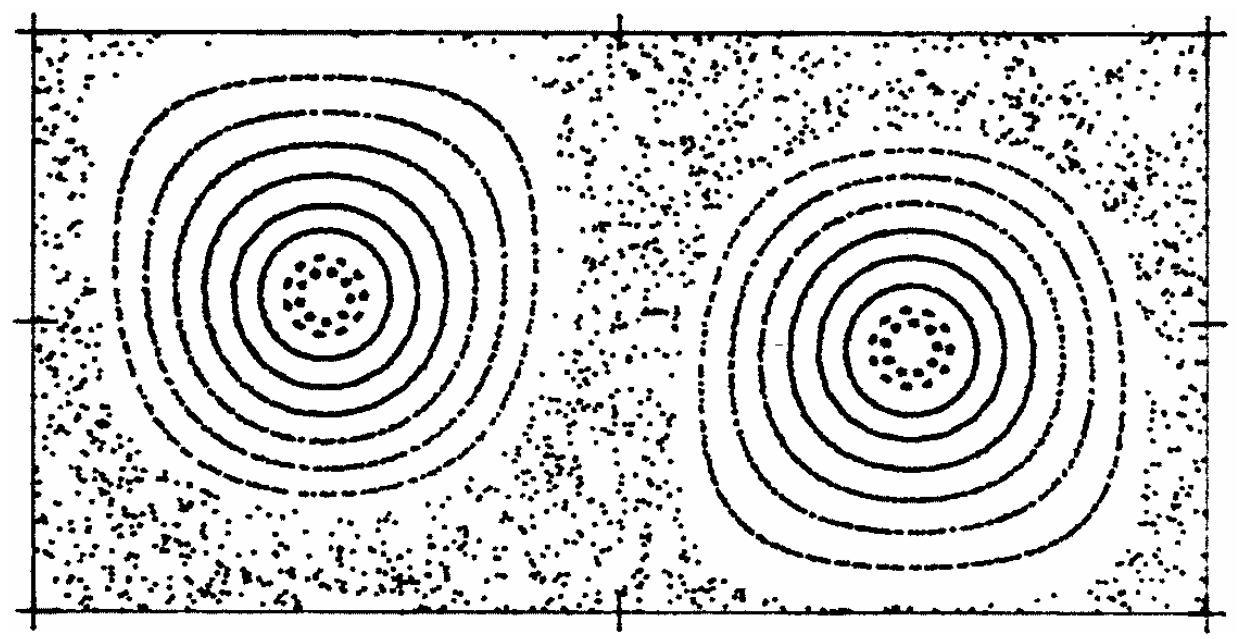

Figure 3. Typical Poincaré section for oscillating vortex flow. Tracers in the central regions of the vortices undergo ordered trajectories, while those near the vortex edges follow chaotic paths.

$2 B / \lambda$ to denote the oscillation amplitude as a fraction of a vortex width.) For the free-slip case,

$$
W(y)=\sin \left(\frac{\pi y}{d}\right)
$$

where $d$ is the vertical extent of the vortex chain. The y-dependence for the no-slip boundary conditions is given by ${ }^{12}$

$$
W(y)=\cos \left(q_{0} y\right)-A_{1} \cosh \left(q_{1} y\right) \cos \left(q_{2} y\right)+A_{2} \sinh \left(q_{1} y\right) \sin \left(q_{2} y\right)
$$

where $\mathrm{q}_{\mathrm{o}}=3.973639, \mathrm{q}_{1}=5.195214, \mathrm{q}_{2}=2.126096, \mathrm{~A}_{1}=0.06151664$ and $\mathrm{A}_{2}=0.103887$. Simulations of particle motion in these flows are conducted by integrating the velocity field using $4^{\text {th }}$-order Runge-Kutta techniques. A typical Poincaré section (Fig. 3) shows the division of the system into ordered regions in the vortex centers and a chaotic sea that wraps around the edges of the vortices ${ }^{3,11}$.

Experimentally, the flow is realized with the use of a magnetohydrodynamic technique $^{13,14}$, as shown in Fig. 4. An electrical current passes through a thin layer of an electrolytic solution (salt water or dilute $\mathrm{H}_{2} \mathrm{SO}_{4}$ ). This current interacts with magnetic fields produced by a chain of alternating polarity magnets, resulting in vortices whose separatrices are located directly above the magnet centers. Time dependence is imposed by displacing the fluid slowly back and forth across the magnet chain with the use of a plunger. Typical frequencies of oscillation are around $0.050 \mathrm{~Hz}$, giving the oscillations a long enough period such that (a) no waves are excited; and (b) the period is significantly longer than the viscous diffusion time ( 4 s).

Transport measurements are conducted by injecting a fluorescent dye (uranine) into the center vortex and illuminating with black-light. For studies with immiscible impurities, a fluorescent oil is used - APD ${ }^{\circledR}$ oil dye P/N 801. A CCD video camera mounted above the apparatus images the system, and these images are digitized on the computer. 
(a)

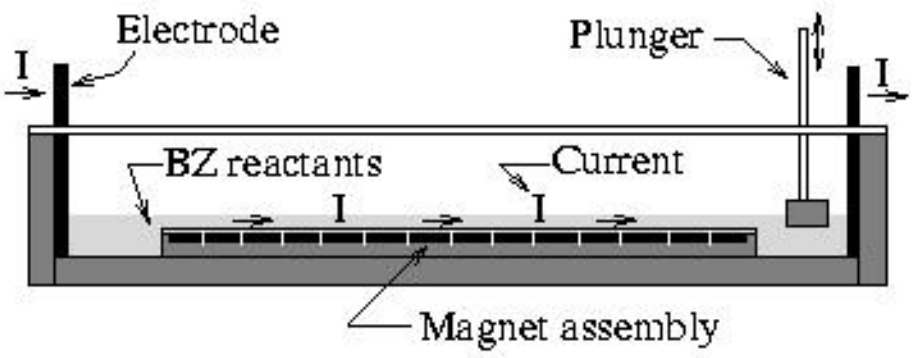

(b)

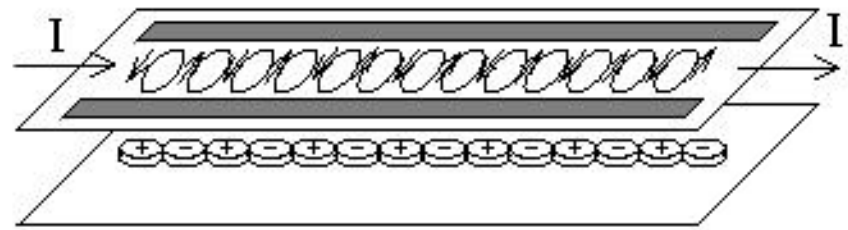

MHD forcing (exploded view)

Figure 4. Experimental apparatus. (a) Side view; (b) exploded view of magnetohydrodynamic forcing. The central region of the flow (where the vortex chain is) measures $3.8 \times 26.7 \mathrm{~cm}$, and the fluid layer is $0.20 \mathrm{~cm}$ thick in this region.

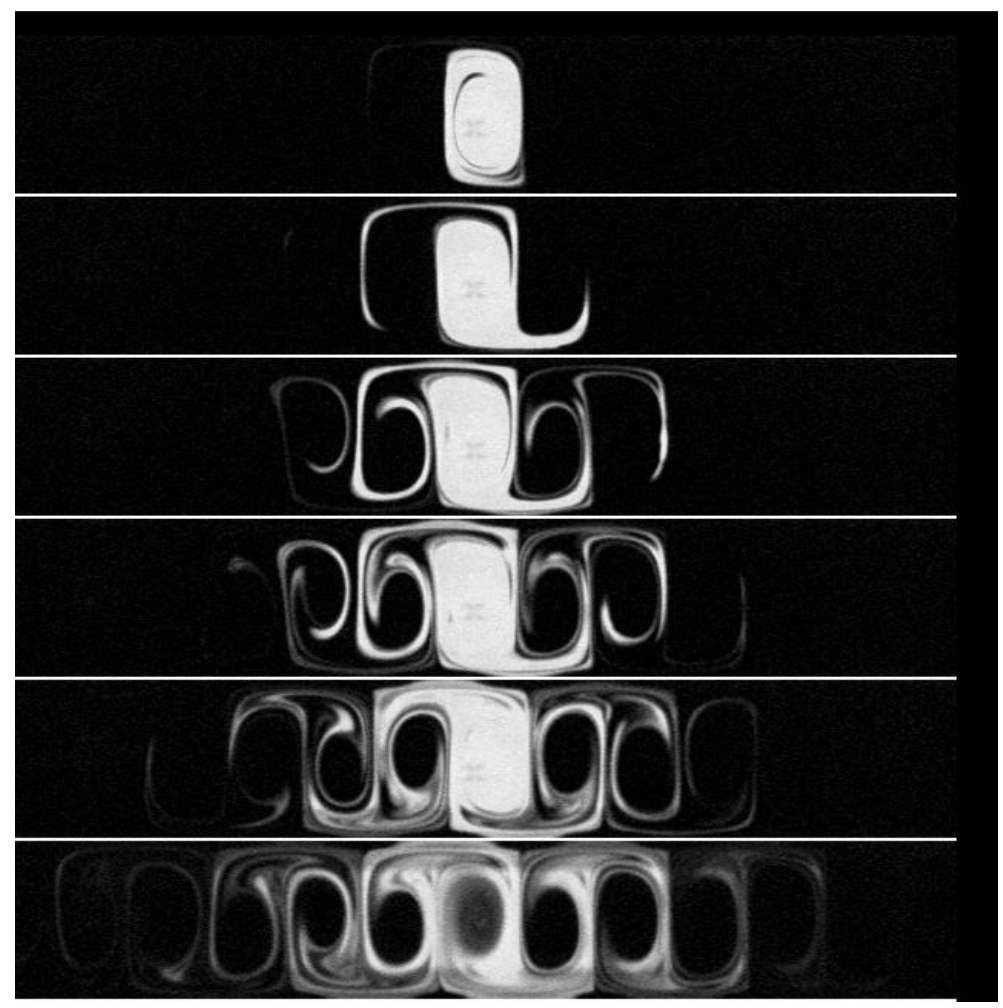

Figure 5. Sequence showing the spreading of a miscible dye in the oscillating vortex chain; the oscillation period is $19 \mathrm{~s}$ and the dimensionless amplitude $b=$ 0.12 . Time from the beginning (from the top): $0,1,2,3,4$, and 10 oscillation periods. 
An example of the spreading of a miscible impurity is shown in Fig. 5. Classic stretching and folding behavior is seen in this sequence, typical of chaotic systems. The division of the system into ordered and disordered regions (similar to those seen in Fig. 3) is manifested in these images by the dark regions in the centers of all the vortices; it takes a long time for dye to penetrate into these regions - mixing into these regions is accomplished only by molecular diffusion and a weak, secondary flow that carries impurities up through the center (as well as clear fluid up through the center of the center vortex $)^{3,15}$. The long-range transport of passive impurities in this system has been studied extensively and is reported on in previous articles. ${ }^{3,4,6}$

For the experiments in which a drop of oil is followed, the computer stores only the coordinates of pixels brighter than a user-defined threshold, enabling us to collect data at a rate of 5-10 images per second for several hours (if the drop stays within the visible region) ${ }^{16,17}$. After the run, the saved coordinates for each frame are re-assembled to determine the $r$ and $\theta$ coordinates of the perimeter of the drop. The elongation $\xi$ can then be determined from the maximum and minimum values of these $r$-coordinates.
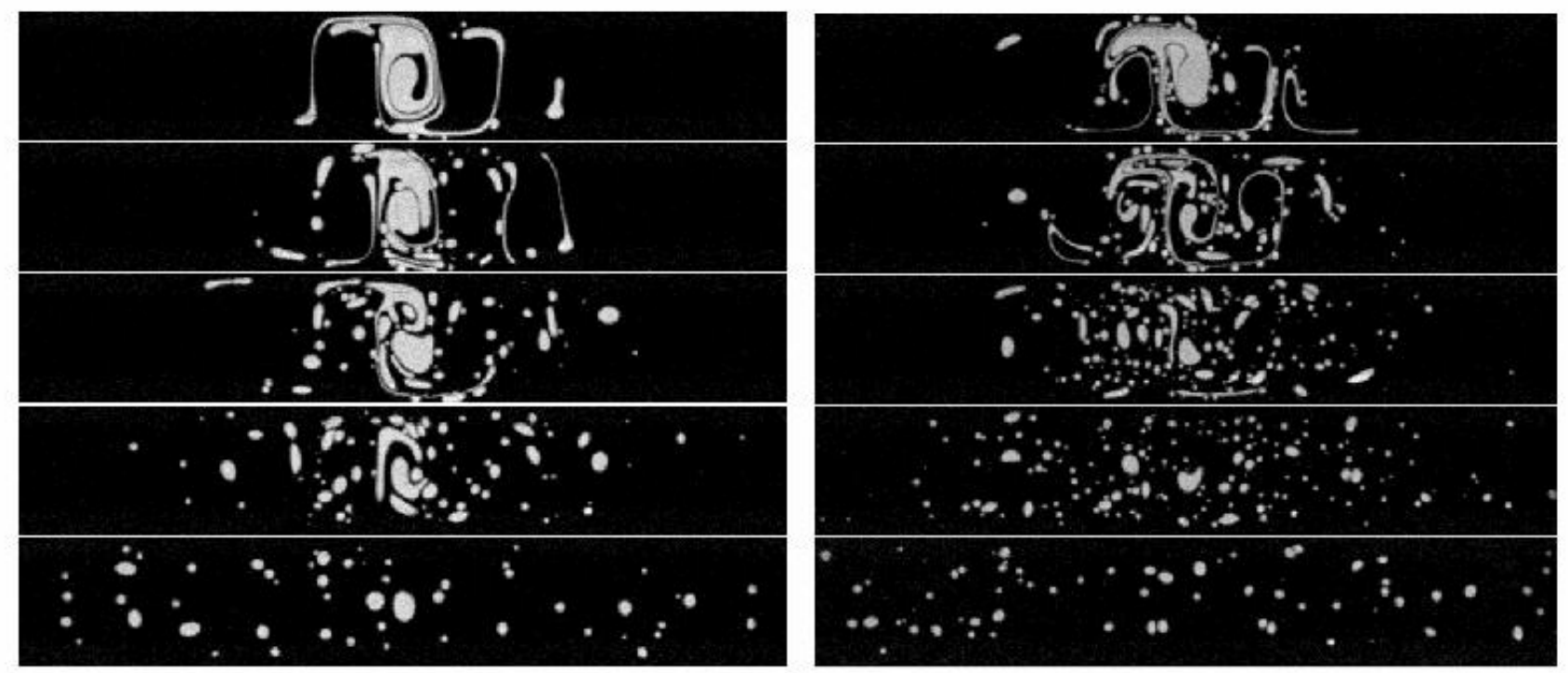

Figure 6. Sequence showing the breakup of oil; $b=0.12$ (left sequence) and $b=0.24$ (right sequence). Time from after start of run (from the top): 18 s, 39 s, 75 s, 189 s, and $1890 \mathrm{~s}$, corresponding to $1,2,4,10$ and 99 periods of oscillation. 


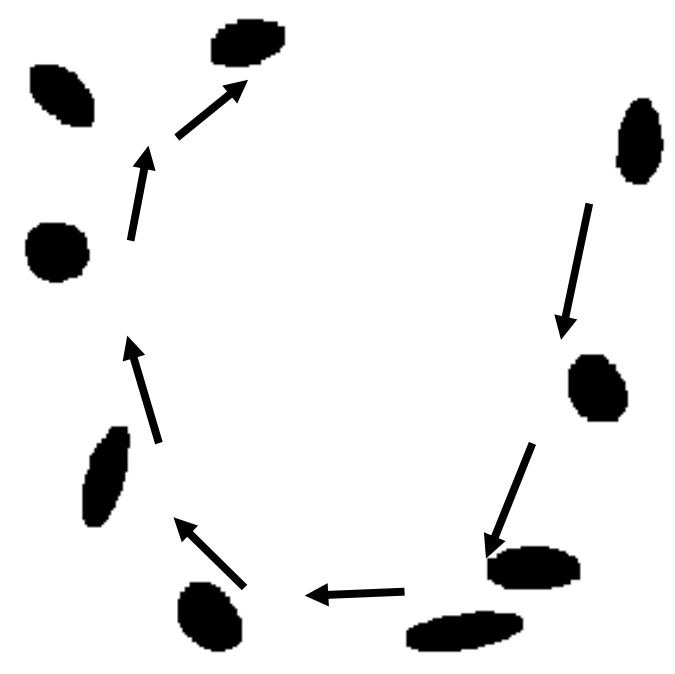

Figure 7. Experimental sequence showing the movement and stretching of a droplet of oil as it is advected around a vortex.

\section{Preliminary experimental and numerical results - drop stretching and breakup}

Experimental evidence of the importance of a Lagrangian framework when considering multiphase processes can be seen by considering the breakup of an immiscible impurity by the oscillating vortex flow ${ }^{14}$ (Fig. 6). In both sequences (columns) of image, the oil initially fills the central vortex but is broken down into a distribution of droplets by the flow. All of the parameters are the same for the two cases except for the amplitude $b$ of the lateral oscillation of the vortex chains. For the case where $b=0.12$ (left column of images), the final steady-state distribution of droplets has a larger average size that for the case with $b=0.24$ (right column); this is a common result where an increase in oscillation amplitude results in smaller droplets in the steady state. With an oscillation amplitude $b=0.06$ (not shown), the final steady-distribution includes significantly larger drops - drops that are typically broken up for larger amplitudes. Clearly, the Eulerian capillary number is insufficient to capture this dependence of the break-up process on the oscillation amplitude, since the velocity gradients in the flow are independent of $b$ with oscillation frequencies as small as those used in these experiments.

The breakup of filaments of oil in a flow with Lagrangian chaos is a complicated problem that will be the subject of future work. Currently, we are exploring the stretching of a single droplet as it is advected through the flow. The sequence in Fig. 7 shows a droplet as it is advected around a vortex. The drop is stretched most as it passes by the hyperbolic fixed points at the corners of the vortex.

The capillary time scale $\tau_{\mathrm{c}}$ is determined with the flow turned off. With the computer acquiring data, we reach into the apparatus with a stick and manually stretch the drop and allow it to relax back while collecting data. This process is repeated several times, resulting in a series of relaxation curves, as shown in Fig. 8. These curves are fit to decaying exponentials of the form $\xi=\xi_{o} \mathrm{e}^{-\mathrm{t} / \tau \mathrm{c}}$, from which a capillary time scale can be determined. 


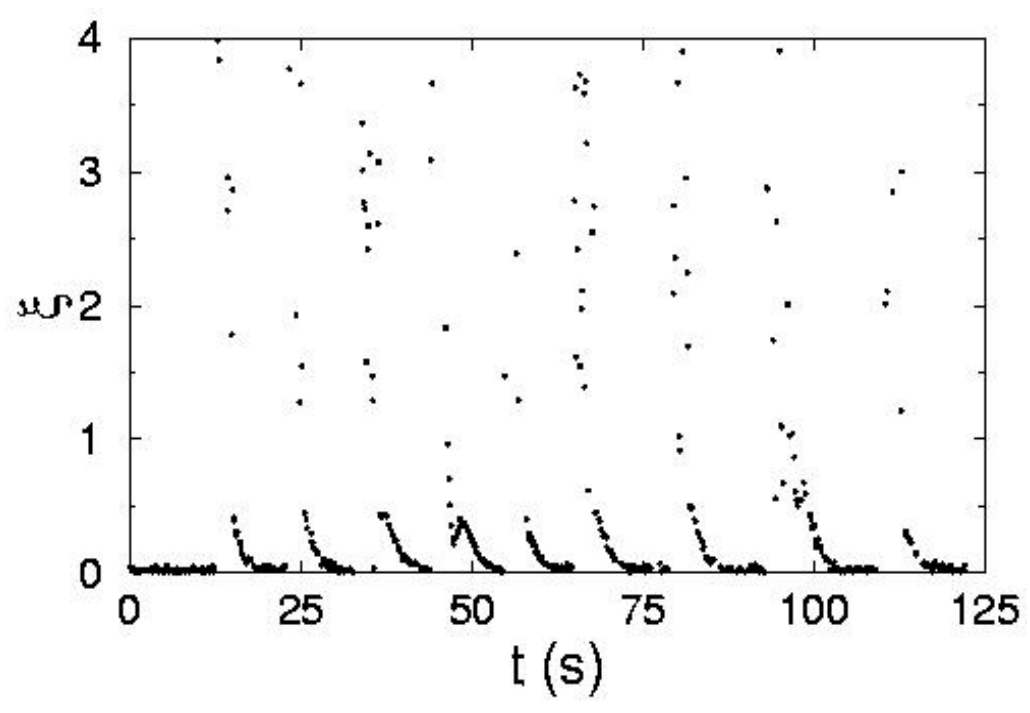

Figure 8. Relaxation of a drop that is repeatedly stretched and then allowed to relax back to a circular shape. These curves (for small elongation $\xi$ ) are fitted to an exponential relation to determine the capillary time scale $\tau_{\mathrm{c}}$.

With the flow turned on, the plots of elongation are more complicated (Fig. 9). For a time-independent flow (no lateral oscillations), there is no Lagrangian chaos, so the oil drop circles around in a closed loop within a single vortex. The trajectory repeats itself, and the drop simply stretches and relaxes periodically, resulting in a plot of $\xi$ that is almost periodic. The resulting probability distribution function (PDF) for the stretch values (Fig. 9b) is a somewhat flat distribution with sharp drop-offs at the minimum and maximum stretch values.

If the flow is time-periodic (with lateral oscillations), then the drop follows a chaotic trajectory if initially placed near the edges of a vortex. In this case, the drop moves around the chaotic region rather than circling on a single closed path. The resulting graph of $\xi$ (Fig. 9c) is significantly different than that for the time-independent case. First, there is much more significant stretching, due to the fact that the drop doesn't just circle in a closed loop; rather the drop samples all of the chaotic region. In particular, the drop occasionally is advected near the hyperbolic fixed points where the velocity gradient is largest. The graph of $\xi$ has significant spikes corresponding to these moments when the drop goes past these fixed points.

The PDF for stretching for the chaotic case is shown in Fig. 9d. The distribution has exponential tails that are (in some cases) asymmetrical. The PDF varies based on the capillary time scale for the drop - Fig. 9d shows PDFs for small (squares) and large (circles) capillary times.

If the definition for the Lagrangian capillary number in Eq. (6) is appropriate, the statistics of the finite-time Lyapunov exponents $\lambda\left(\tau_{c}\right)$ experienced by a tracer moving through the flow should mimic the statistics in Fig. 9. Numerically-determined maps of $\lambda\left(\tau_{\mathrm{c}}\right)$ for the oscillating vortex flow are shown in Fig. 10 for three different oscillation amplitudes and four different time scales. In the long-time limit, $\lambda\left(\tau_{c} \rightarrow \infty\right)$ reduces to the largest standard Lyapunov 

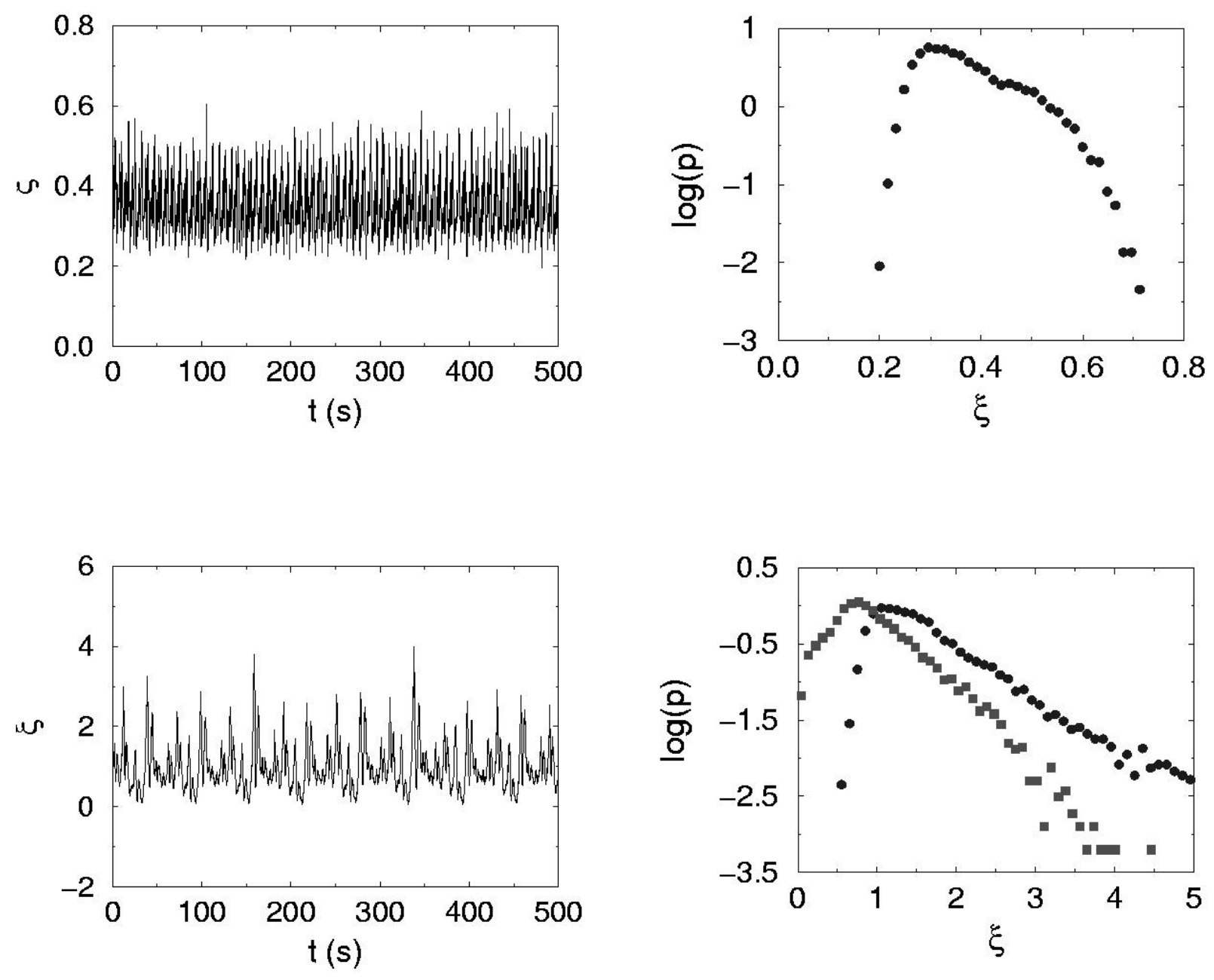

Figure 9. Drop elongation in the presence of a flow. (a) Time-series of elongation $\xi$ for time-independent flow $(b=0)$; (b) probability distribution function (pdf) corresponding to (a); (c) time-series of elongation $\xi$ for time-periodic flow with $b=0.12$; (d) pdf corresponding to (c). The squares in (d) correspond to $\tau_{\mathrm{c}} \approx 1 \mathrm{~s}$ and the circles correspond to $\tau_{\mathrm{c}}=3 \mathrm{~s}$.

exponent, which is a positive constant in the chaotic region and 0 in the ordered region. In this limit (bottom row in Fig. 10), the maps of finite-time Lyapunov exponents resemble the Poincaré section for this flow (compare with Fig. 3).

We simulate the motion of a passive tracer through the flow, noting the value of $\lambda\left(\tau_{c}\right)$ at each point along its motion. From these values, we can determine a PDF for $\lambda\left(\tau_{\mathrm{c}}\right)($ Fig. $11 \mathrm{a})$. Multiplying $\lambda\left(\tau_{c}\right)$ by the capillary time scale $\tau_{c}$ gives us the Lagrangian-generalized capillary number $C_{L}$ defined in Eq. 6 . The PDF showing the statistics for the resulting $C_{L}$ experienced by a tracer moving through the flow are shown in Fig. $11 \mathrm{~b}$ and c. Note the qualitative similarity 
between Fig. 11c and Fig. 9d; in particular, we find exponential tails and asymmetrical distributions, similar to what we found for the experimental PDFs of the stretching $\xi$ of the drop in the flow. This is consistent with the proposal that the Lagrangian capillary number is the appropriate quantity for describing drop stretching.

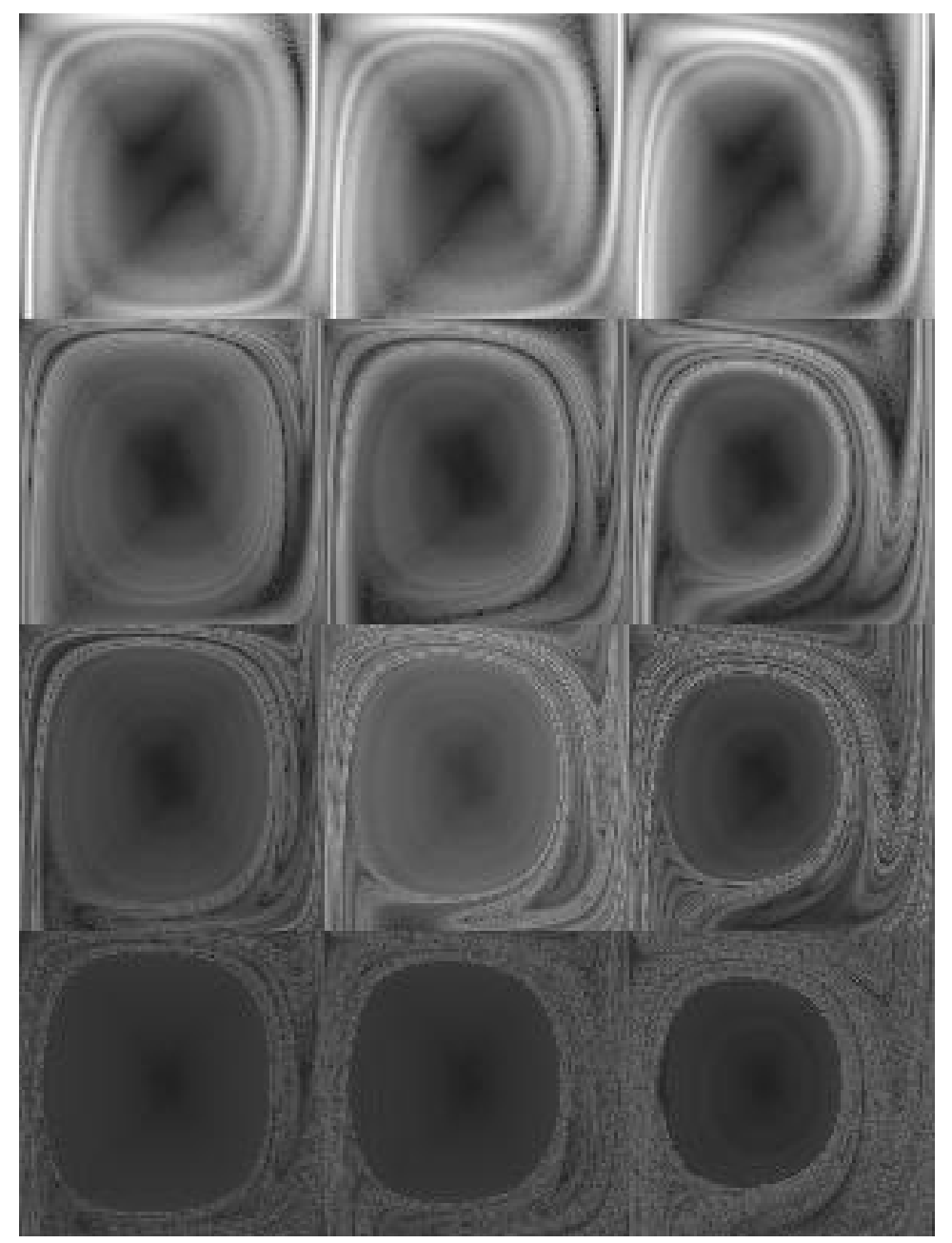

Figure 10. Maps of finite-time Lyapunov exponents for the oscillating vortex flow; $b=0.06$ (left column), $\mathrm{b}=0.12$ (middle column) and $\mathrm{b}=0.24$ (right column). Non-dimensional time-scales for the maps are (from the top down): 2, 5, 10 and 25.

\section{On-going work - the blinking vortex flow.}

There are some issues that complicate experimental verification of the Lagrangian capillary number with the oscillating vortex flow. Most significant is the finite size of the drop, especially considering the limited size of the chaotic region for the oscillating vortex flow (see Fig. 3). As a result, the drop is not always completely within the chaotic regime. In fact, it is quite likely that the drop in these studies frequently overlaps the ordered and chaotic regions.

With this in mind, we have plans to study droplet stretching in a second system to complement the oscillating vortex flow studies. The flow that will be used is the blinking vortex 
flow, motivated by Aref's 1984 paper on chaotic advection ${ }^{1}$. The flow and the apparatus used to study this flow are shown in Fig. 12. A cylindrical container holds a shallow layer of an electrolytic solution. Two point electrodes (denoted by “+” in Fig. 12a) are located off-center along the center line of the cell, and a ring electrode surrounds the fluid. The entire apparatus sits on top of a 2.5 " diameter Nd-Fe-Bo magnet which produces a uniform magnetic field throughout the region of interest. Current flows radially outward, alternating periodically between the two center electrodes. The result is a flow that rotates alternately around the left and right center electrodes.
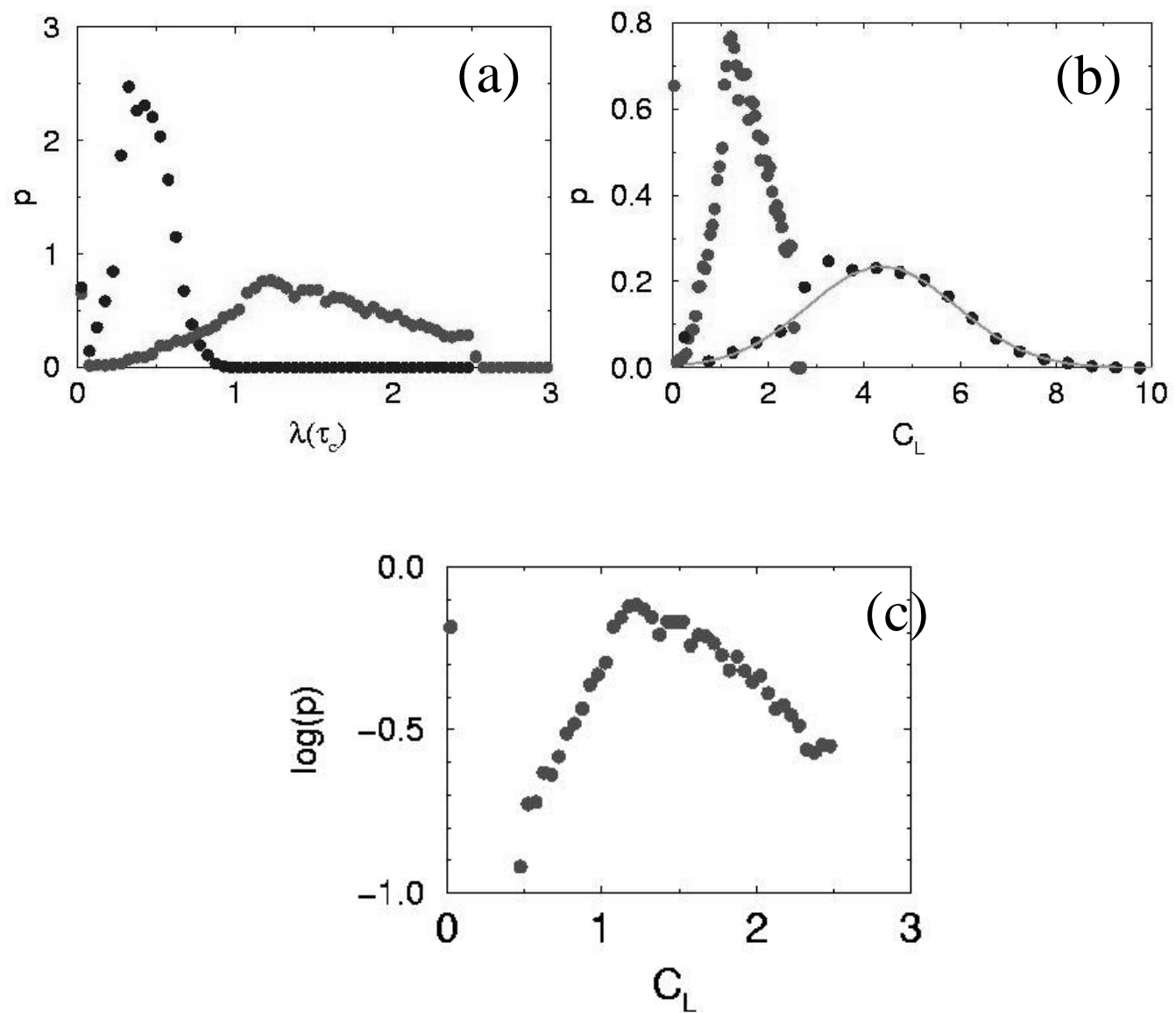

Figure 11. Results of numerical simulations of statistics of finite-time Lyapunov exponents and Lagrangian capillary number. (a) Probability distribution functions (PDFs) of finite-time Lyapunov exponents; $\tau_{\mathrm{c}}=10$ (left curve), $\tau_{\mathrm{c}}=1$ (right curve). (b) PDF for Lagrangian capillary number $C_{L}=\tau_{\mathrm{c}} \lambda\left(\tau_{\mathrm{c}}\right) ; \tau_{\mathrm{c}}=1$ (left curve), $\tau_{\mathrm{c}}=10$ (right curve). (c) Enlargement of PDF for $C_{L}$ for $\tau_{\mathrm{c}}=1$.

Poincaré sections for the blinking vortex flow (see Ref. 1) reveal a chaotic region that covers the center region of the flow. In conjunction with the fact that this system is significantly larger than the oscillating vortex flow, it is our expectation that it will be possible to track oil drops in the flow that remain entirely within the chaotic region.

Preliminary tests have been conducted to verify that the experimental system does, in fact, have chaotic mixing like Aref's blinking vortex flow. The advection of a line of fluorescent 


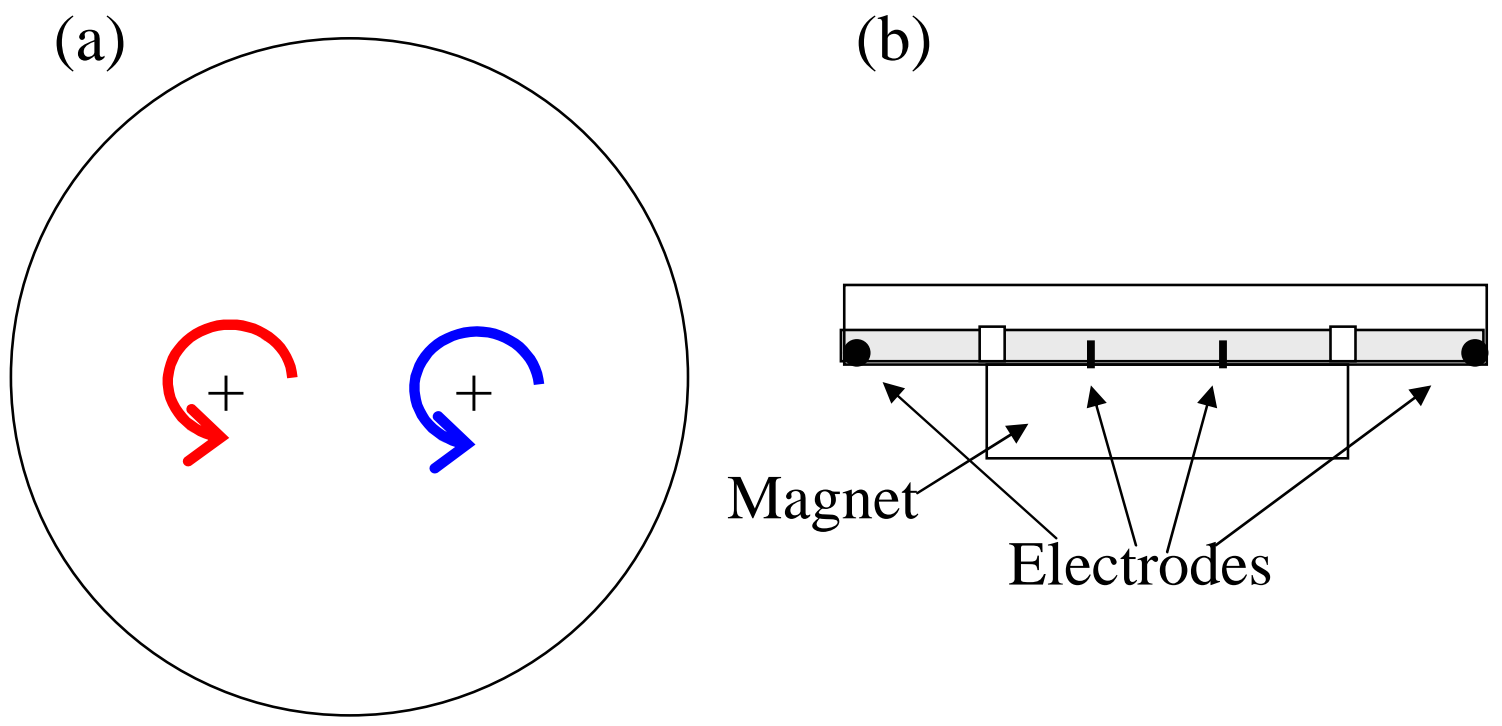

Figure 12. Blinking vortex flow. (a) Sketch of flow. (b) Side view of apparatus that generates the flow.

dye in this flow is shown in Fig. 13. The classic stretching and folding seen in this figure verifies that mixing is chaotic in this system, as expected based on the results of Aref's 1984 simulations.

We are currently studying the stretching of oil in this blinking vortex flow. The results of these studies will be the subject of a future article.
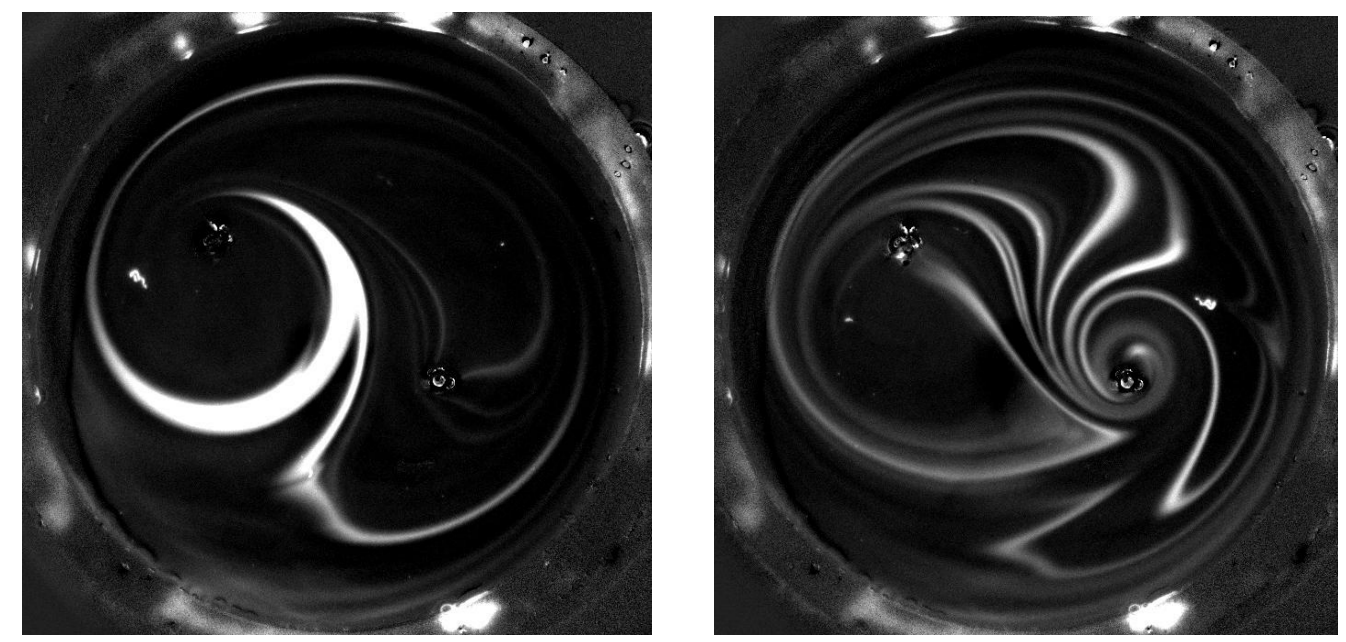

Figure 13. Mixing of dye in the blinking vortex flow; oscillation period $\mathrm{T}=20 \mathrm{~s}$. The time after injection of dye is $35 \mathrm{~s}$ (1.8 periods of oscillation) for the left image and $60 \mathrm{~s}$ (3 periods of oscillation) for the right image. 


\section{Discussion and conclusions}

In this article, we have argued that the behavior of a drop in a flow should be examined from a Lagrangian perspective, which is the natural framework since it follows the motion of the drop. This is particularly important in light of the fact that trajectories for tracers can be chaotic even for laminar, well-ordered flows. We have proposed a generalization of the capillary number that accounts for the advection of a drop through the flow and the varying shear experienced by that drop. We have done some preliminary tests of this idea in an oscillating vortex flow, comparing the statistics of the stretching $\xi$ of a drop with those of the Lagrangian capillary number $C_{L}$. The preliminary results were encouraging - probability distribution functions of both $\xi$ and of $C_{L}$ for drops in a chaotic region have asymmetrical exponential tails.

Although it is clear that a Lagrangian generalization of the capillary number is needed, much more work needs to be done to determine if the particular form proposed in Eq. 6 is the most appropriate form. More experimental runs are needed with a wide variety of flow parameters. Data that will be obtained with the blinking vortex flow will also help establish the limits of validity of the proposed Lagrangian capillary number for drop stretching.

If the capillary number is sufficiently large, the drop will break-up. Studies are currently in progress to assess whether there is a critical value of $C_{L}$ above which advected drops can be expected to break. We are also investigating the opposite process; namely coarsening of a distribution of initially-small droplets into larger drops in a flow. 


\section{Acknowledgements}

We are pleased to acknowledge useful discussions with Jean-Luc Thieffault, Igor Mezic and Howard Stone, as well as the assistance of Dave Vayda in the construction of the apparatus. The research was supported by the US National Science Foundation (grants DMR-0071771 and PHYS-0097424).

\section{References}

${ }^{1}$ Aref, H., J. Fluid Mech. 143, 1 (1984).

${ }^{2}$ Ottino, J.M., Leong, C. W., Rising, H. , and Swanson, P. D. , Nature 333, 419 (1988).

${ }^{3}$ Solomon, T. H. , and Gollub, J. P., Phys. Rev. A 38, 6280 (1988).

${ }^{4}$ Camassa, R., and Wiggins, S., Phys. Rev. A 43, 774 (1991).

${ }^{5}$ Solomon, T.H., Tomas, S., and Warner, J. L., Phys. Rev. Lett. 77, 2682 (1996).

${ }^{6}$ Solomon, T.H., Lee, A.T., and Fogleman, M.A., Physica D 157, 40 (2001).

${ }^{7}$ Castiglione, P., Crisanti, A., Mazzino, A., Vergassola, M., and Vulpiani, A., J. Phys. A 31, 7197 (1998).

${ }^{8}$ J.M. Rallison, J.M., Annu. Rev. Fluid Mech. 16, 45 (1984).

${ }^{9}$ Stone, H.A., Annu. Rev. Fluid Mech. 26, 65 (1994).

${ }^{10}$ Stone, H.A., and Leal, L.G., J. Fluid Mech. 206, 223 (1989); Milliken, W.J., and Leal, L.G., J. Non-Newtonian Fluid Mech. 40, 355 (1991).

${ }^{11}$ Gollub, J. P., and Solomon, T. H., Phys. Scripta 40, 430 (1989).

${ }^{12}$ Chandrasekhar, S., Hydrodynamic and Hydromagnetic Stability, Dover (New York), 1961.

${ }^{13}$ Willaime, H., Cardoso, O., and Tabeling, P., Phys. Rev. E 48, 288 (1993).

${ }^{14}$ Solomon, T.H., Tomas, S., and Warner, J.L., Phys. Fluids 10, 342 (1998).

${ }_{15}^{15}$ Solomon, T.H., Transport and Boundary Layers in Rayleigh-Bénard Convection, $\mathrm{PhD}$ thesis, 1990.

${ }^{16}$ Pervez, M.S., and Solomon, T.H., Exp. Fluids 17, 135 (1994).

${ }^{17}$ Solomon, T.H., Weeks, E.R., and Swinney, H.L., Phys. Rev. Lett. 71, 3975 (1993). 